\title{
Entrenamiento para la mejora de disfunciones atencionales en niños y adolescentes con Síndrome de Asperger a través de estimulación cognitiva directa
}

\author{
María-Ángeles Bravo-Álvarez* y María Frontera-Sancho \\ Departamento de Psicología y Sociología. Universidad de Zaragoza. Zaragoza (España).
}

\begin{abstract}
Resumen: Esta investigación analiza la capacidad de generalización del aprendizaje en niños y adolescentes con Síndrome de Asperger a aspectos cognitivos atencionales con demandas diferentes a las ejercitadas mediante la utilización de un programa de estimulación cognitiva directa. La muestra estuvo constituida por 15 casos con Síndrome de Asperger entre 7 y 15 años $(M=12 ; D T=2.7)$ con dificultades atencionales, medidas a través de instrumentos de orientación psicométrica, a los que se aplicó un programa de entrenamiento en atención dos horas semanales durante seis meses consecutivos. Los resultados del análisis pretest-postest mostraron diferencias estadísticamente significativas en todos los subsistemas atencionales. En atención selectiva las diferencias son de magnitud moderada en las variables relacionadas con la velocidad de procesamiento, en mantenida el tiempo de reacción disminuyó considerablemente (magnitud de la diferencia moderada) y aumentaron los aciertos disminuyendo los errores (ambos con una magnitud de diferencia grande). En atención alternante se observó un mayor número de aciertos, y en dividida se obtuvieron mayores niveles de respuestas totales (los dos de magnitud moderada). Se concluye que el rendimiento atencional en los casos analizados mejora tras la aplicación de un programa de entrenamiento cognitivo específico siendo capaces de generalizar el aprendizaje a medidas psicométricas.
\end{abstract}

Palabras Clave: Disfunciones Atencionales; Síndrome de Asperger; Terapia Cognitiva Específica; Programa Attention Game.

\section{Introducción}

La investigación que presentamos analiza la capacidad de generalización del aprendizaje a medidas psicométricas (Van Zomeren y Fasotti, 1992), a través del entrenamiento con terapia cognitiva específica, en niños y adolescentes con Síndrome de Asperger (SA) que muestran déficits de atención. El trastorno del espectro autista (TEA) es un trastorno del neurodesarrollo que se caracteriza por déficits significativos en la interacción social y comunicación, e intereses y conductas restrictivas y repetitivas (APA, 2013). En esta línea continua del TEA, el SA (OMS, 1992) se sitúa en la zona superior del espectro, representando la parte menos severa y con mayores habilidades cognitivas y de lenguaje.

La clínica del SA hace referencia a interacciones sociales inadecuadas, dificultad para utilizar e interpretar correctamente las claves sociales, comunicación y uso del lenguaje peculiares (gestualidad y entonación inadecuada, habla pedante y tangencial, empleo abusivo del turno de palabra conversación "unilateral”), gusto por la repetición de ciertas conductas e intereses restringidos. Bajo estas peculiaridades subyacen, además, determinados aspectos cognitivos entre los que se sitúan los problemas de atención que, si no de manera universal, muy frecuentemente son observados en SA y marcan un estilo de aprendizaje y funcionamiento coti-

* Dirección para correspondencia [Correspondence address]

María-Ángeles Bravo-Álvarez. Facultad de Educación. Universidad de Zaragoza. C/ Pedro Cerbuna 12. 50009 Zaragoza (España).

E-mail: marian@unizar.es
Title: Training to improve attentional dysfunction in children and adolescents with Asperger Syndrome through direct cognitive stimulation.

Abstract: This research analyzes the generalization ability of learning in children and adolescents with Asperger Syndrome to attentional cognitive aspects with different demands on exercised through the use of a direct cognitive stimulation program. The sample consisted of 15 cases with Asperger between 7 and 15 years $(M=12, S D=2.7)$ with attentional difficulties, measured by psychometric orientation instruments, to which an attention training program was implemented to two hours per week for six consecutive months. The results of the pretest-posttest analysis showed statistically significant differences in all attentional subsystems. Selective attention differences are moderate in variables related to processing speed, in sustained attention the reaction time significantly decreased (moderate magnitude of the difference) and increased the successes reducing errors (both with a large magnitude difference). In alternating attention, a greater number of successes were observed and in split attention higher levels of total responses (both moderate magnitude) were obtained. It is concluded that the attentional performance in the cases analyzed improvement after applying a cognitive training program specifically being able to generalize learning psychometric measures.

Key words: Attentional Dysfunction; Asperger Syndrome; Specific Cognitive Therapy; Attention Game Program.

diano (Asperger, 1944; Belinchón, Hernández y Sotillo, 2009; Frontera-Sancho, 2010).

Los trabajos que analizan déficits atencionales específicos de los perfiles clínicos dentro de los TEA, específicamente SA y autismo de alto funcionamiento (AAF), señalan posibles déficits en los cuatro componentes atencionales propuestos por el modelo de Mirsky (1989, 1996): excitación, orientación y filtrado; inhibición de respuestas prepotentes; atención sostenida; y capacidad para el cambio atencional.

Concretamente, Rogers y Ozonoff (2005) realizan una revisión de las teorías sobre activación señalando que existen más pruebas que avalan un estado cercano a una hiporeactividad estimular en los subgrupos de nivel intelectual más alto. Tanto en SA como en AAF las respuestas de alerta y orientación son más lentas que en desarrollo neurotípico, disminuyendo significativamente la velocidad de desacoplamiento (Belinchón, Hernández y Sotillo, 2008; Landry y Bryson, 2004). Respecto a los problemas de inhibición de respuestas prepotentes los resultados no son concluyentes. Mientras que en algunos estudios no se obtuvieron conclusiones claras a favor del déficit en este tipo de tareas (Belinchón et al., 2009); otros señalan dificultades el grupo con SA, así como preferencia local frente a global (Rinehart, Bradshaw, Moss, Brereton y Tonge, 2006). Estas dificultades están relacionadas con déficits atencionales en focalización y codificación; es decir, dificultades para concentrar los recursos atencionales en una tarea específica mientras se realiza una tarea o alguna operación cognitiva sobre ella, eliminando distractores del entorno. 
Las investigaciones que examinan posibles dificultades de atención sostenida muestran argumentos a favor del déficit tanto a nivel auditivo (Casey, Gordon, Mannheim y Rumsey, 1993), como en tareas de vigilancia visual (Goldstein, Johnson y Minshew, 2001; López-Frutos, Sotillo, Tripicchio y Campos, 2011; Schatz, Weimer y Trauner, 2002). A su vez, numerosas investigaciones coinciden en señalar la presencia de dificultades para el cambio atencional en las tareas que requieren flexibilidad cognitiva y rapidez de respuesta (Courchesne et al., 1994; Goldstein et al., 2001; Ozonoff, South y Miller, 2000; Pascualvaca, Fantie, Papageorgiu y Mirsky, 1998; Plaisted, Swettenham y Rees, 1999). Los estudios que han explorado la aparente naturaleza de este déficit, lo asocian a problemas en los cambios atencionales «estratégicos» relacionados con tareas propias de la función ejecutiva (Jarocci, Burack, Shore, Mottron y Enns, 2006; Ozonoff et al., 2000).

Existe, por tanto, un consenso unánime sobre la frecuente presencia de alteraciones atencionales en individuos con SA. No obstante, la gran heterogeneidad que caracteriza al síndrome, es también observada en aspectos cognitivos atencionales comparando individuos con el mismo diagnóstico (Bravo-Álvarez, Frontera-Sancho y Herrero-Nivela, 2012). La ausencia de un perfil atencional universal en los niños con SA hace preciso, de cara a una posible intervención, la aplicación de un programa adaptado a las peculiaridades cognitivas del síndrome que se adecúe individualmente a cada sujeto.

La rehabilitación cognitiva a través de la Terapia Cognitiva Específica o Estimulación Cognitiva directa o dirigida, ha estado disponible y ha resultado exitosa en individuos con otros trastornos neurológicos (Bernabeu-Verdú et al., 2004; Butler y Copeland, 2002; Hendriks y Van der BroekSandmann, 1996; López-Luengo, Fernández, Losada y García, 2002). Parte del supuesto de que la estimulación específica, con entrenamiento en áreas cognitivas concretas, es un requisito esencial en la recuperación de la función (Robertson, 1999). En estos programas se requiere que cada área del proceso cognitivo esté fundamentada en un modelo teórico. Las tareas deben administrarse repetidamente para facilitar el crecimiento o la regeneración neuronal y así la función podría mejorarse (Powell, 1981), y las metas y objetivos deben organizarse jerárquicamente para facilitar una administración sistemática. Esta jerarquización exige que los procesos cognitivos complejos se dividan en un número limitado de conductas específicas de modo que, cuando se alcanza el objetivo en un ejercicio se debe pasar a tareas de nivel superior.

Existe un claro acuerdo entre investigadores sobre la eficacia de los tratamientos de rehabilitación cognitiva través de la Terapia Cognitiva Específica respecto a la mejoría en las tareas entrenadas a través de ejercicios de repetición y práctica estructurada; sin embargo, aparecen discrepancias en los resultados si se trata de evaluar la efectividad del tratamiento y su generalización tanto en el tiempo como en otras actividades que las tareas se diferencian más de las que se utilizan en la rehabilitación (Gray, Robertson, Pentland y Anderson, 1997; Malec, Rao, Jones y Stubbs, 1984; Ponsford y Kinsella, 1988; Sturm, Wilmes, Orgass y Hartje, 1987; Wood, 1986).

Pese a la aplicación de cuantiosos programas destinados a la mejorar las habilidades de pensamiento, no encontramos estudios en los que se hubiera aplicado a niños y adolescentes con SA un programa específico de entrenamiento en habilidades atencionales que cumpliera los requisitos de los programas de Terapia cognitiva específica, adaptado a sus peculiaridades cognitivas. En función de estas consideraciones, nuestro estudio tiene como objetivo evaluar la capacidad de generalización del aprendizaje a actividades en las que las tareas requieran del ejercicio de aspectos cognitivos atencionales con demandas diferentes a las ejercitadas utilizando un programa de estimulación cognitiva directa, diseñado ex profeso de acuerdo a las peculiaridades cognitivas del SA y AAF, al que hemos denominado Attention Game (AG).

AG es un programa de intervención informatizado de aplicación individual a partir de ocho años cuyo objetivo es el entrenamiento de funciones de atención en cuatro subprocesos atencionales: atención selectiva, atención mantenida, atención alternante y atención dividida. Se ha simplificado la complejidad estimular, se presenta la información con frases directas y breves con soporte visual a través de un Agente Pedagógico (AP) informatizado y el entrenamiento es dirigido por un responsable del entrenamiento que favorece un aprendizaje sin errores (Bravo-Álvarez, 2014). Tanto el AP como el responsable del entrenamiento realizan una supervisión y ofrecen feedback permanentes utilizando el refuerzo contingente y entrenamiento en estrategias (Ponsford, Sloan y Snow, 1995). El entrenamiento es de complejidad creciente, con diferentes estímulos y combinando entre ejercicios con diferentes modalidades sensoriales visuales y auditivas (Cappa, Benke, Clarke, Rossi, Stemmer y Van Heugten, 2005; Cicerone et al., 2000).

\section{Método}

\section{Participantes}

Los participantes fueron seleccionados entre aquellos procedentes de la Asociación Aragonesa Asperger con diagnóstico confirmado según criterios del DSM IV-TR. Sobre todas las solicitudes de participación en el estudio, se seleccionaron aquellos que demandaban intervención por déficits atencionales (déficits observados por padres y/o profesores), no estaban incluidos en ninguna otra categoría diagnóstica de trastorno mental asociado, y no recibieran o hubieran recibido tratamiento en rehabilitación de disfunciones atencionales. La muestra quedó formada por 15 niños y adolescentes con edades comprendidas entre los 7 y 15 años, con una media de edad de 12 años ( $D S=2.7)$ de los que un $93.33 \%$ eran varones $(n=14)$ y $6.67 \%$ mujeres $(n=1)$. Todos los participantes se encuentran escolarizados en centros ordinarios sin adaptaciones curriculares significativas. El cociente intelectual de los participantes, valorado a través de la escala 
de inteligencia WISC IV, presenta una oscilación de valores entre 74 y $116(M=88, D S=11.5)$, situándose más de $60 \%$ entre puntuaciones de 81 a 89 .

\section{Procedimiento e Instrumentos}

La batería de exploración neuropsicológica estaba formada por instrumentos de orientación psicométrica destinados a la valoración de atención selectiva, mantenida, alternante y dividida. Con el objetivo de medir la atención selectiva fueron administradas las subpruebas Claves y Búsqueda de Símbolos de la escala de inteligencia de Wechsler para niños (WISC-IV) y el Test Stroop, se recogieron los "aciertos" de las dos subpruebas del WISC-IV y la variable "resistencia a la interferencia” en el test Stroop (Golden, 2001). Atención mantenida fue valorada a través del test Children Sustained Attention Task (CSAT) registrando "aciertos, errores y el tiempo de reacción” (Servera y Llabrés, 2004). Seleccionamos la variable "aciertos" del Trail Making Test, parte B, para evaluar atención alternante (Reynolds, 2002) y atención dividida fue medida mediante el test AGL (Blanca, Zalabardo, Rando, López-Montiel y Luna, 2005), recogiendo el "número total de respuestas" y la diferencia entre el mayor número de respuestas y el menor de la proporción "procesamiento global y local" (variable que hemos denominado "mayor-menor").

La batería de exploración neuropsicológica fue administrada un mes previo al tratamiento, en dos sesiones de hora y media aproximadamente. Al mes de finalizar el tratamiento, planificado y supervisado por la misma psicóloga que realizaba las evaluaciones, se volvió a administrar las citadas pruebas. El programa de estimulación cognitiva directa se implementó individualmente por psicopedagogos adiestrados en el manejo del programa.

En nuestro estudio, el tratamiento consistió en la ejecución de ejercicios de atención mantenida, selectiva, alternante y dividida, regulados por niveles de dificultad de acuerdo a las normas de aplicación del programa Attention Game (Apéndice I). Cada participante realizó un total de 32 sesiones de tratamiento durante seis meses, a razón de dos sesiones semanales, de una hora aproximada de duración.

\section{Análisis de datos}

Se realizó una comparación intragrupal mediante la prueba no paramétrica de los rangos con signo Wilcoxon en aras de identificar posibles diferencias entre los cambios cognitivos atencionales antes y después del tratamiento, con un nivel de significación de $p<.05$. Las probabilidades de la prueba de Wilcoxon se acompañaron de medidas de tamaño del efecto en el caso de apreciarse diferencias estadísticamente significativas entre los dos momentos de la comparación. $\mathrm{Al}$ respecto, se utilizó el parámetro delta de Cliff $(\delta C)$ (Cliff, 1993) con la intención de complementar la significación estadística de una medida no paramétrica de magnitud (Fernández y Fernández, 2009).

\section{Resultados}

Los resultados de la comparativa antes y después del tratamiento mediante la prueba de Wilcoxon muestran diferencias estadísticamente significativas en todos los subsistemas atencionales con una magnitud de la diferencia moderada o grande según variables (Tabla 1).

Tabla 1. Prueba de Wilcoxon sobre las variables de la batería de exploración neuropsicológica antes y después del tratamiento.

\begin{tabular}{|c|c|c|c|c|c|c|c|c|}
\hline & \multirow[t]{2}{*}{ Variable } & \multicolumn{2}{|c|}{ Antes } & \multicolumn{2}{|c|}{ Después } & \multirow[t]{2}{*}{$Z$} & \multirow[t]{2}{*}{$p$} & \multirow[t]{2}{*}{$\delta C^{a}$} \\
\hline & & $M$ & DT & $M$ & DT & & & \\
\hline \multirow{3}{*}{ Atención Selectiva } & Búsqueda de Símbolos & 21.60 & 10.59 & 83.60 & 13.96 & $-3,205$ & $.001 * * *$ & -.37 \\
\hline & Claves & 36.80 & 10.57 & 14.53 & 11.18 & $-3,186$ & $.001 * * *$ & -.50 \\
\hline & Interferencia & 1.19 & 7.10 & 3.64 & 7.18 & $-1,109$ & .267 & \\
\hline \multirow[t]{3}{*}{ Atención mantenida } & Aciertos & 65.60 & 21.20 & 83.60 & 13.96 & $-3,112$ & $.002^{* *}$ & -.49 \\
\hline & Errores & 32.40 & 30.20 & 14.53 & 11.18 & $-2,951$ & $.003^{* *}$ & .46 \\
\hline & Tiempo de reacción & 356.67 & 36.92 & 336.20 & 51.65 & $-2,018$ & $.044^{*}$ & .39 \\
\hline Atención alternante & Aciertos & 20.27 & 3.58 & 22.33 & 2.09 & $-2,536$ & $.011^{*}$ & -.40 \\
\hline \multirow[t]{2}{*}{ Atención dividida } & Mayor-Menor & 11.47 & 15.86 & 4.80 & 4.66 & $-1,480$ & .139 & \\
\hline & Total de respuestas & 35.13 & 16.06 & 46.53 & 19.37 & $-3,065$ & $.002^{* *}$ & -.34 \\
\hline
\end{tabular}

Nota: ${ }^{\text {a Tamaño del efecto calculado si } p<.05 ; p<.01 ; p<.001}$

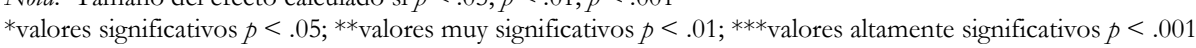

Concretamente, en atención selectiva encontramos diferencias estadísticamente significativas en las variables relacionadas con velocidad de procesamiento de la información, con una magnitud de la diferencia moderada en Búsqueda de símbolos $(Z=-3,205 ; p=.001 ; \delta C=-.37)$ y de magnitud grande en Claves $(Z=-3,186 ; p=.001 ; \delta C=-.50)$.

En todas las variables relacionadas con atención mantenida aparecieron diferencias estadísticamente significativas: se redujo de manera significativa el tiempo de reacción a los estímulos con una magnitud moderada $(Z=-2,018 ; p=.044$; $\delta C=.39)$, la media del $\mathrm{n}^{\circ}$ de aciertos aumentó reduciéndose entre ambos valores la desviación típica con una diferencia estadísticamente significativa de magnitud grande $(Z=$ $3,112 ; p=.002 ; \delta C=-.49)$. La media de errores y la desviación típica disminuyeron resultando una diferencia estadísticamente significativa de magnitud grande $(Z=-2,951 ; p=$ .003 ; $\delta C=.46$ ). La comparativa entre las dos mediciones indica que tras el tratamiento se cometieron significativamente 
menos errores y más aciertos con menor tiempo de reacción a los estímulos.

En atención alternante se obtuvieron también diferencias estadísticamente significativas de magnitud moderada $(Z=$ $2,536 ; p=.011 ; \delta C=-.46)$, y en atención dividida se apreciaron diferencias estadísticamente significativas significativa de magnitud moderada en la cantidad de respuestas totales $(Z=$ $-3,065 ; p=.012 ; \delta C=-.34)$ no ocurriendo así en la variable “mayor-menor". No obstante, en ésta última variable, pese a no observarse diferencias estadísticamente significativas, se aprecia una reducción de la diferencia tras el tratamiento lo que supone una mejor ejecución en atención dividida ya que se han aplicado los dos procesamientos (local y global) conjuntamente en superior proporción.

\section{Discusión y conclusiones}

Los datos obtenidos en la muestra estudiada en la evaluación pre-test en los cuatro subsistemas atencionales (atención mantenida, selectiva, alternante y dividida) coinciden con los reportados en la literatura al señalar dificultades en la velocidad de procesamiento y respuestas más lentas de alerta y orientación en personas con SA (Belinchón et al., 2008). Se observa un rendimiento inferior en tareas de vigilancia visual tal y como señalan investigaciones que comparan individuos con SA grupos sin alteraciones de desarrollo (Goldstein et al., 2001; López-Frutos et al., 2011; Schatz et al., 2002). Al mismo tiempo, los resultados obtenidos en las variables relacionadas con atención alternante y dividida muestran un déficit en las tareas de cambio a nivel conceptual (Jarocci et al, 2006).

La comparativa de los resultados obtenidos antes y después del tratamiento mostró que los participantes incluidos en el estudio presentaban una mejora estadísticamente significativa en variables de atención selectiva relacionadas con velocidad de procesamiento, en atención mantenida el tiempo de reacción ante estímulos disminuyó considerablemente, en atención alternante se observó un mayor número de aciertos, y en atención dividida obtuvimos mayores niveles de respuestas totales. El tamaño de la diferencia es una verdadera medida de la significancia de tal diferencia (Morales, 2012) $y$, por tanto, es un dato de especial relevancia que en seis meses, en todos los subsistemas atencionales se aprecien magnitudes de las diferencias moderadas o grandes.

Tal y como hemos señalado, no existen discrepancias entre investigadores sobre la eficacia de los tratamientos de rehabilitación cognitiva respecto a la mejoría en las tareas entrenadas a través de ejercicios de repetición y práctica estructurada; sin embargo, aparecen divergencias en los resultados cuando se evalúa la efectividad del tratamiento y su generalización tanto en el tiempo como en otras actividades que las tareas se diferencian más de las que se utilizan en la rehabili- tación (Gray, Robertson, Pentland y Anderson, 1997; Malec, Rao, Jones y Stubbs, 1984; Ponsford y Kinsella, 1988; Sturm, Wilmes, Orgass y Hartje, 1987; Wood, 1986). Si bien, Park y Ingles (2001) y Park y Barbuto (2005) señalan que estos cambios pueden deberse a la práctica de tareas entrenadas con el programa de rehabilitación cognitivo y no a la recuperación de la atención específicamente; otros señalan que las actividades funcionales que pueden controlarse voluntariamente y con implicación de atención por parte del sujeto han de ser trabajadas mediante el entrenamiento específico en esa actividad funcional (Cappa et al., 2005).

Los principales hallazgos de nuestra investigación indican que los participantes incluidos en el estudio presentaban una mejora significativa en aquellos ejercicios que requieren de aspectos cognitivos similares a los entrenados con el programa pero con demandas diferentes a las ejercitadas. Pese a que no existe evidencia suficiente para realizar una recomendación única sobre las estrategias que se deben utilizar en la rehabilitación neuropsicológica de la atención, nuestros datos muestran argumentos a favor de una generalización del aprendizaje a medidas psicométricas.

Las personas con SA presentan dificultades a la hora de abstraer los principios que subyacen a la resolución de problemas (Martín-Borreguero, 2004) quedándose con lo específico y manifestando grandes obstáculos para generalizar el aprendizaje a situaciones nuevas. Bajo esta peculiaridad que caracteriza el perfil cognitivo del síndrome, cualquier intervención que potencie la generalización del aprendizaje ofrece muestras de su efectividad y, por tanto, los programas de entrenamiento cognitivo directo pueden suponer importantes beneficios para las dificultades atencionales que frecuentemente presenta este tipo de población.

A pesar de las implicaciones de esta investigación para la comprensión y avance en el tratamiento de disfunciones atencionales en $\mathrm{SA}$, los resultados deben ser interpretados en el contexto de una serie de limitaciones. Este estudio se caracteriza por un pequeño tamaño de la muestra no probabilístico apropiado para un primer estudio de viabilidad; sin embargo, también restringe las inferencias respecto a la generalización de los resultados. La condición de grupo control no se incluyó debido a la imposibilidad de equiparar grupos de los participantes que fueron incluidos en la investigación de acuerdo a variables de edad, perfil atencional, cociente intelectual y contextual, dada la variabilidad característica del propio síndrome. La ausencia de la condición de grupo control limita, por tanto, las inferencias relativas a la especificidad de los efectos del tratamiento con programas de entrenamiento cognitivo directo. Así, quedan abiertos interrogantes que apuntan nuevas líneas de estudio sugiriendo aumentar tanto la duración del tratamiento como el número de participantes, incluyendo la condición de grupo control. 


\section{Referencias}

American Psychiatric Association (2013). Diagnostic and Statistical Manual of Mental Disorders (DSM V). Arlington: American Psychiatric Publishing. doi: 10.5555/appi.books.9780890425596.x00pre

Asperger, H. (1944). Die «Autistischen Psychopathen» im Kindesalter. Archiv für Psychiatrie und Nervenkrankheiten, 117, 76-136. doi:10.1007/BF01837709

Bernabeu-Verdú, J., López-Luengo, B., Fournier-del Castillo, C., CañeteNieto, A., Suárez-Rodríguez, J., y Castel-Sánchez, V. (2004). Aplicación del Attention Process Training dentro de un proyecto de intervención en procesos atencionales en niños con cáncer. Revista de Neurología, 38, 473-482. Recuperado de http:/ / www.revneurol.com/sec/resumen.php?or=web\&i=e\&id=2003 $\underline{571 \#}$

Belinchón, M., Hernández, J. M., y Sotillo, M. (2008). Personas con Síndrome de Asperger: Funcionamiento, detección y necesidades [OX Reader version]. Recuperado de https://www.uam.es/centros/psicologia/paginas/cpa/paginas/doc/d ocumentacion/personasconsindromedeasperger2008.pdf

Belinchón, M., Hernández, J. M., y Sotillo, M. (2009). Síndrome de Asperger: una guía para los profesionales de la educación [OX Reader version]. Recuperado de https://www.uam.es/centros/psicologia/paginas/cpa/paginas/doc/d ocumentacion/guiaparaprofesionales.pdf

Blanca, M. J., Zalabardo, C., Rando, B., López-Montiel, D. y Luna, R. (2005). AGL, Atención Global-Local. Madrid: TEA Ediciones.

Bravo-Álvarez, M., Frontera-Sancho, M. y Herrero-Nivela, M. L. (2012). Perfil Atencional en niños con Síndrome de Asperger. Revista de Psicopatología y Salud Mental del niño y del adolescente, 20, 29-38. Recuperado de http://www.fundacioorienta.com/index.php/article/index/170

Bravo-Álvarez, M. (2014). Efectividad del programa "Attention Game" en el tratamiento de disfunciones atencionales en trastorno de espectro autista (sindrome de Asperger) (tesis doctoral no publicada). Universidad de Zaragoza: Zaragoza.

Butler, R. W. y Copeland, D. R. (2002). Attentional processes and their remediation in children treated for cancer: a literature review and development of a therapeutic approach. J Int Soc Neuropsychol, 8, 115-24. doi:10.1017/S1355617701020112

Cappa, S. F., Benke, T., Clarke, S., Rossi, B., Stemmer, B. y Van Heugten, C. M. (2005). EFNS guidelines on cognitive rehabilitation: report of an EFNS task force. Eur J Neurol, 12, 665-80. Recuperado de http://almacengpc.dynalias.org/publico/Rehabilitacion $\% 20$ cognitiva $\% 202005$.pdf

Casey, B., Gordon, C., Mannheim, G. y Rumsey, J. (1993). Dysfunctional attention in autistic savants. Journal of Clinical and Experimental Neuropsychology, 15(6), 933-946. doi: 10.1080/01688639308402609

Cicerone, K. D., Dahlberg, C., Kalmar, K., Langenbahn, D. M., Malec, J. F., Bergquist, T. F. ...Morse, P. A. (2000). Evidence-based cognitive rehabilitation: recommendations for clinical practice. Arch Phys Med Rehabil, 81, 1596-615. doi: http://dx.doi.org/10.1053/apmr.2000.19240

Cliff, N. (1993). Dominance statistics: ordinal analyses to answer ordinal questions. Psychological Bulletin, 114(3), 494-509. doi: 10.1037/00332909.114.3.494

Courchesne, E., Townsend, J., Akshoomoff, N., Saitoh, O., YeungCourchesne, R., Lincoln, A. J., ... Lau, L. (1994). Impairment in shifting attention in autistic and cerebellar patients. Behavioral Neuroscience, 108(5), 848-865. doi: 10.1037//0735-7044.108.5.848

Fernández, A. y Fernández, I. M. (2009). Críticas y alternativas a la significación estadística en el contraste de hipótesis. Madrid: La Muralla.

Frontera-Sancho, M. (2010). Funcionamiento cognitivo en síndrome de Asperger. En Federación Asperger España Síndrome de Asperger (Eds), Aspectos discapacitantes y Valoración [OX Reader version]. Recuperado de http://www.asperger.es/publicaciones.php/

Golden, C. J. (2001). Test Stroop de colores y palabras. Madrid: TEA Ediciones.

Goldstein, G., Johnson, C. y Minshew, N. (2001). Attentional processes in autism. Journal of Autism and Developmental Disorders, 31(4), 433-440. doi: 10.1023/A:1010620820786
Gray, J. M., Robertson, I., Pentland, B. y Anderson, S. (1992). Microcomputer-based attentional retraining after brain damage: a randomized group controlled trial. Neuropsychol Rehabil, 2, 97-115. doi: 10.1080/09602019208401399

Hendriks, C. M. y Van der Broek-Sandmann, T. M. (1996). Amsterdamse training van aandacht en geheugen vorr kinderen (ATAG-K). Amsterdam: Harcourt Test Publisher.

Jarocci, G., Burack, J., Shore, D., Mottron, L. y Enns, J. (2006). Globallocal visual processing in high functioning children with Autism: Structural vs. implicit task biases. Journal of Autism and Developmental Disorders, 36(1), 117-129. doi: 10.1007/s10803-005-0045-2

Landry, R. y Bryson, S. E. (2004). Impaired disengagement of attention in young children with autism. Journal'of'Child Psychology and Psychiatry, 45, 1115-1122. doi: 10.1111/j.1469-7610.2004.00304.x

López-Frutos, J. M., Sotillo, M., Tripicchio, P. y Campos, R. (2011). Funciones atencionales de orientación espacial, alerta y control ejecutivo en personas con trastornos del espectro autista. Revista de Psicopatología y Psicología Clínica, 16(2), 101-112. Recuperado de http://www.aepcp.net/arc/02_2011_n2_frutos_sotillo_tripicchio_cam pos.pdf

López-Luengo B., Fernández S., Losada M. L. y García M. L. (2002). Programa de rehabilitación de las capacidades atencionales en pacientes con demencia tipo Alzheimer y enfermedades cerebrovasculares. Mapfre Medicina, 13, 186-96. Recuperado de http://sid.usal.es/idocs/F8/8.2.6-6098/programa.pdf

Malec, J., Rao, N., Jones, R. y Stubbs, K. (1984). Video game practice effects on sustained attention in patients with craniocerebral trauma. Cognit Rehabil, 2, 18-23. Recuperado de http://psycnet.apa.org/index.cfm?fa=buy.optionToBuy\&id=1991 10520-001

Martín-Borreguero, P. (2005). Perfil lingüístico del individuo con síndrome de Asperger: implicaciones para la investigación y la práctica clínica. Revista de Neurología, 41 (Supl 1), 115-122. Recuperado de http://www.sld.cu/galerias/pdf/sitios/rehabilitacion$\underline{\log / \text { perfil linguistico de asperger.pdf }}$

Mirsky, A. F. (1989). The neuropsychology of attention: elements of a complex behavior. En E. Perecman (Ed), Integrating theory and practice in clinical neuropsychology (pp. 75-91). Hillsdale, N.J.: Erlbaum.

Morales, P. (2012). El tamaño del efecto (effect siže): análisis complementarios al contraste de medias. Recuperado de http://www.upcomillas.es/personal/peter/investigacion/Tama $\% \mathrm{~F} 1 \mathrm{O}$ DelEfecto.pdf

Organización Mundial de la Salud (1992). Clasificación de Trastornos Mentales y de Conducta: Descripciones Clínicas y Guías de Diagnóstico (CIE-10). Ginebra: OMS.

Ozonoff, S., South, M. y Miller, J. (2000). DSM-IV-defined Asperger syndrome: Cognitive, behavioral and early history differentiation from high-functioning autism. Autism, 4(1), 29-46. doi: $10.1177 / 1362361300041003$

Park, N. W. y Barbuto, E. (2005). Treating attention impairments. En P. W. Halligan y D. T. Wade (Eds.), Effectiveness of rehabilitation for cognitive deficits (pp. 81-90). Oxford: Oxford University Press.

Park, N. W. y Ingles, J. L. (2001). Effectiveness of attention rehabilitation after an acquired brain injury: a meta-analysis. Neuropsychology, 15, 199$210 . \quad$ Recuperado

https://www.apa.org/pubs/journals/releases/neu152199.pdf

Pascualvaca, D., Fantie, B., Papageorgiu, M. y Mirsky, A. (1998). Attentional capacities in children with autism: Is there a general deficit in shifting focus? Journal of Autism and Developmental Disorders, 28, 467-478. doi: 10.1023/A:1026091809650

Plaisted, K., Swettenham, J. y Rees, L. (1999). Children with autism show local precedence in a divided attention task and global precedence in a selective attention task. Journal of Child Psychology and Psychiatry, 40, 733$742 . \quad$ Recuperado de http://journals.cambridge.org/action/displayAbstract? fromPage $=$ onli ne\&aid $=10867 \&$ fileId $=$ S0021963099004102 doi: $10.1111 / 1469$ 7610.00489 
Ponsford, J. y Kinsella, G. (1988). Evaluation of a remedial programme for attentional deficits following closed-head injury. J Clin Exp Neuropsychol, 10, 693-708. doi: 10.1080/01688638808402808

Ponsford, J., Sloan, W. y Snow, P. (1995). Traumatic brain injury: rehabilitation for everyday adapting living. Hove, UK: Lawrence Erlbaum.

Powell, G.E. (1981). Brain Function Therapy. Hants: Gower-Aldershot.

Reynolds, C.R. (2002). Comprehensive Trail Making Test: Examiner's manual. Austin, Texas: PRO-ED.

Rinehart, N., Bradshaw, J., Moss, S., Brereton, A. y Tonge, B. (2006). Pseudo-random number generation in children with high-functioning autism and Asperger's disorder. Autism, 10(1), 70-85. doi: $10.1177 / 1362361306062011$

Robertson, I. (1999). Theory-driven neuropsycological rehabilitation: the role of attention and competition in recovery of function after brain damage. En D. Gopher y A. Koriat (Eds), Attention and Performance XVII: Cognitive Regulation of Performance: Interaction of Theory and Application (pp. 677-696). Cambridge, MA: MIT Press.

Rogers, S. J. y Ozonoff, S. (2005). What do we know about sensory dysfunction in autism? A critical review of the empirical evidence. Journal of Child Psychology and Psychiatry, 46(12), 1255-1268. doi: 10.1016/i.trd.2012.05.006

Schatz, A., Weimer, A. y Trauner, D. (2002). Brief Report: Attention differences in Asperger Syndrome. Journal of Autism and Developmental Disor ders, 32(4), 333-336. doi: 10.1023/A:1016339104165

Servera, M. y Llabrés, J. (2004). CS AT: Tarea de atención sostenida en la infancia. Madrid: TEA Ediciones.

Sturm, W., Wilmes, K., Orgass, B. y Hartje, W. (1997). Do specific attention deficits need specific training? Neuropsychol Rehabil, 7, 81-103. Recuperado http://www.limef.com/Downloads/Sturm $\% 20$ et $\% 20 \mathrm{al} \% 20$ NeuroReh ab.pdf

Van Zomeren, H. A. y Fasotti, L. (1992). Impairments of attention and approaches to rehabilitation. En O. Von Cramon, E. Póppel, y N. Von Steinbúchel (Eds), Brain damage and rehabilitation: a neuropsychological approach (pp. 23-51). Heidelberg: Springer-Verlag.

Wood, R.L. (1986). Rehabilitation of patients with disorders of attention. J Head Trauma Rehabil, 3, 43-53. doi: 10.1097/00001199-198609000$\underline{00008}$

(Articulo recibido: 06-01-2015; revisado: 27-03-2015; aceptado: 12-0-2015) 


\section{Apéndice I}

El programa AG es un programa de aplicación individualizada, que trabaja a través de una serie de ejercicios atencionales que estimulan los siguientes ámbitos de la atención:

Atención mantenida (con ejercicios que entrenan en tareas de vigilancia y de rendimiento continuo), Atención selectiva (con ejercicios que entrenan en tareas de focalización y discriminación), Atención alternante (con ejercicios que entrenan en tareas de cambio atencional), y Atención dividida (con ejercicios que entrenan en tareas de concentración y de capacidad).

Hemos denominado a cada una de estas funciones atencionales "subsistemas atencionales". Attention Game reorganiza los ejercicios en cada subsistema atencional diferenciando distintas áreas (engloban una habilidad cognitiva atencional general) de las que se desprenden una o varias dimensiones (especifican la habilidad cognitiva del área a la que pertenecen) que contienen niveles diferentes. La estructura general del programa puede observarse en la figura 1.

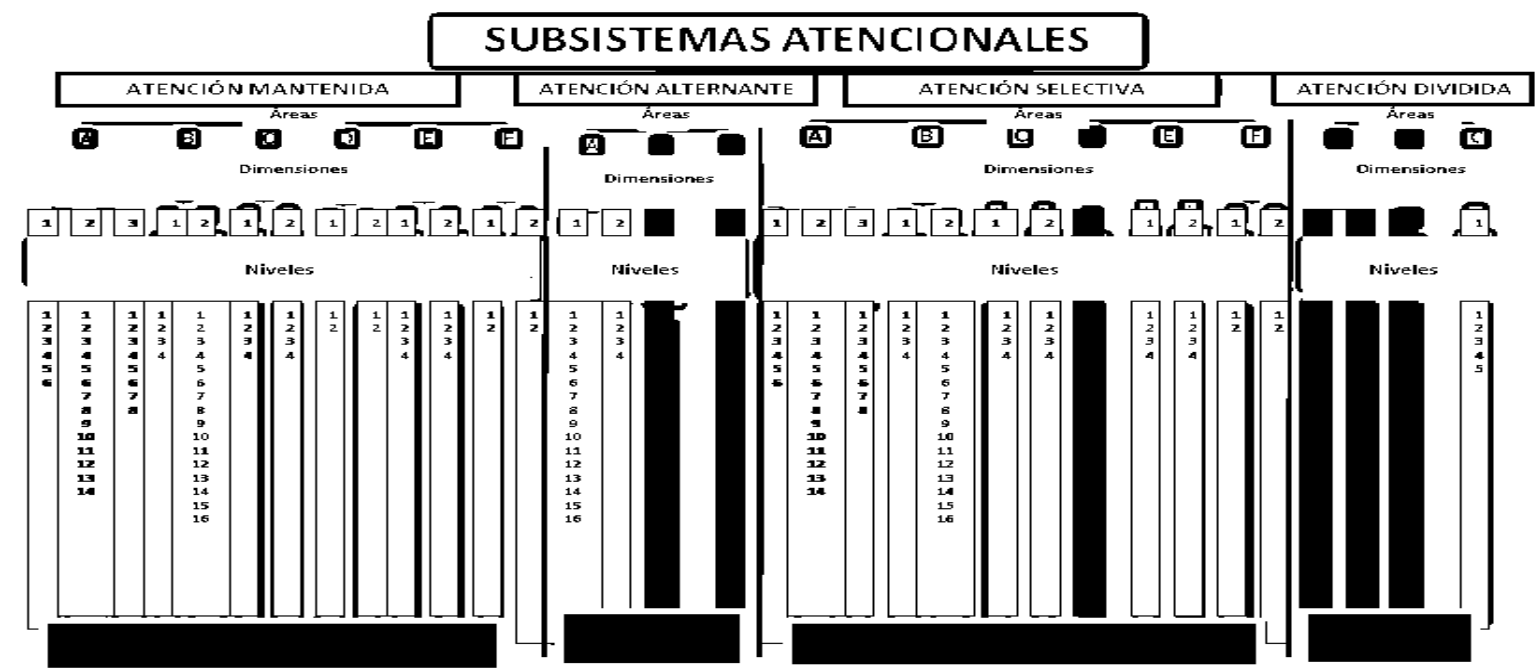

Figura 1. Estructura general del programa Attention Game.

Los niveles de cada dimensión contienen los ejercicios a realizar. En cada nivel, se presenta el ejercicio UNA VEZ a modo de prueba. Finalizada la prueba, volverá a presentarse el ejercicio para su resolución. Para superar un nivel y poder practicar el siguiente, debe realizarse correctamente DOS veces y de forma consecutiva el ejercicio propuesto. El entrenamiento a través de los diferentes ejercicios o niveles se ha trazado jerárquicamente de acuerdo a dos criterios entrelazados: a) presentación visual/auditiva, y b) presentación estimular "lenta"/"rápida". Se entrena primero ejercicios que tengan soporte visual con un tiempo de presentación estimular ("lenta"). Continúa el entrenamiento con ejercicios de soporte visual en los que se reduce el tiempo de presentación estimular ("rápida"). Posteriormente, ejercicios con soporte auditivo con un tiempo de presentación estimular ("lenta") y finalmente se entrenan ejercicios con soporte auditivo en los que se reduce el tiempo de presentación estimular ("rápido").

Antes de comenzar el entrenamiento es necesario realizar un test de nivel (figura 2). Esta prueba supone la adaptación personalizada del entrenamiento al indicar aquellos subsistemas atencionales en los que tiene mayor o menor dificultad según el número de niveles superados. Finalizado el test, las sesiones de entrenamiento se programarán comenzando y finalizando con aquellos subsistemas en los que mostró menor dificultad. 


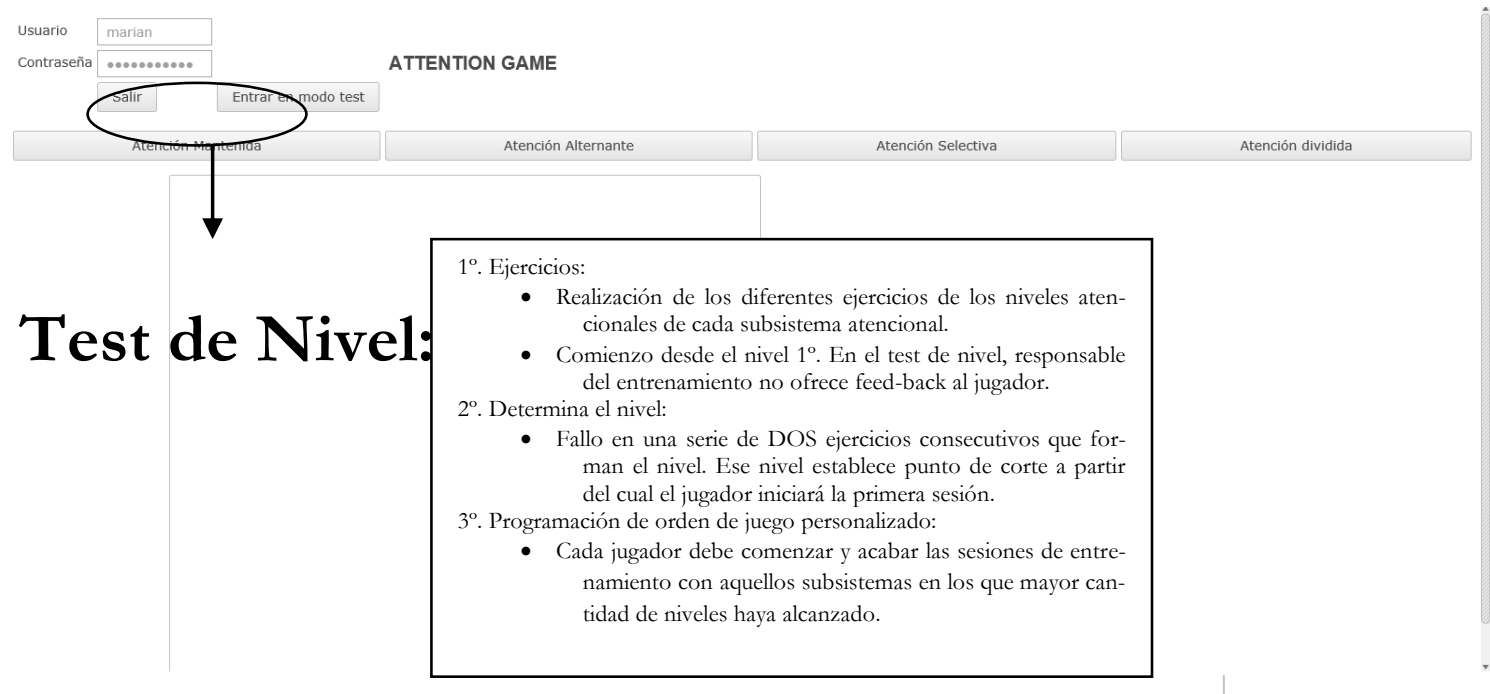

Figura 2. Test de nivel.

En cada sesión de entrenamiento (aproximadamente de una hora) deben trabajarse ejercicios de cada subsistema atencional de acuerdo al orden programado según el test de nivel. Cada sesión de entrenamiento sigue una pauta específica: primero se explica en qué consiste la atención y qué se logra cuando ésta no funciona correctamente (Aprendizaje Metacognitivo). Los niveles de cada dimensión contienen un ejercicio que explica el agente pedagógico informatizado (AP) y el responsable del entrenamiento. El jugador puede realizarlo a modo de prueba una vez pudiendo en esta ocasión ofrecer al jugador todo tipo de feed-back para asegurar un aprendizaje sin errores. Finalizada la prueba, el AP indica el comienzo del entrenamiento. Cada ejercicio se repite hasta que se realiza correctamente dos veces consecutivas sin cometer más errores de los permitidos. Durante el proceso, el responsable del entrenamiento observa cómo se realiza el ejercicio a fin de detectar el motivo de los errores y el poder ayudarle a que lo haga de forma correcta. A su vez, el AP indica si los errores cometidos son de omisión o comisión. Con el objetivo de facilitar la generalización de lo aprendido a contextos naturales, la intervención se completa con una serie de instrucciones relacionadas con actividades de la vida cotidiana relacionadas con el contenido de lo ejercitado. 